06

\title{
Температурная динамика доменной структуры триглицинсульфата по данным атомно-силовой микроскопии и диэлектрической спектроскопии
}

\author{
(С) Г.И. Овчинникова ${ }^{1}$, Н.В. Белугина ${ }^{2}$, Р.В. Гайнутдинов ${ }^{2}$, Е.С. Иванова ${ }^{2}$, \\ В.В. Гребенев ${ }^{2}$, А.К. Лашкова ${ }^{2}$, А.Л. Толстихина ${ }^{ף, 2}$ \\ ${ }^{1}$ Московский государственный университет им. М.В. Ломоносова, \\ Москва, Россия \\ ${ }^{2}$ Институт кристаллографиии им. А.В. Шубникова РАН, \\ Москва, Россия \\ ๑ E-mail: alla@ns.crys.ras.ru
}

\begin{abstract}
Представлены результаты комплексного исследования водородсодержащего сегнетоэлектрика триглицинсульфата (TGS) методами атомно-силовой микроскопии (AFM) и диэлектрической спектроскопии. В режиме микроскопии пьезоэлектрического отклика (PFM) in situ изучена динамика доменной структуры при нагреве и охлаждении кристалла TGS вблизи фазового перехода. Получены релаксационные зависимости общего периметра доменных границ и размеров доменов. Измеренные в диапазоне частот от 10 до $10^{11} \mathrm{~Hz}$ диэлектрические спектры TGS проанализированы на основе представлений о заметном вкладе проводимости в диэлектрический отклик сегнетоэлектриков. Показано, что учет вклада проводимости в диэлектрические спектры дает хорошее согласие с экспериментом и позволяет получить дополнительную информацию о температурной динамике доменной структуры.
\end{abstract}

Работа выполнена с использованием оборудования ЦКП ИК РАН при поддержке Министерства образования и науки.

\section{1. Введение}

В настоящее время не существует единой точки зрения относительно закономерностей эволюции доменной структуры и ее связи с основными макроскопическими параметрами (поляризация, диэлектрическая проницаемость и др.) кристалла TGS. В большинстве случаев доменную динамику сопоставляют с температурным поведением низкочастотной диэлектрической проницаемости $\varepsilon(T)$, справедливо считая, что она отражает динамику доменной структуры [1-3]. Однако эти исследования не дают полного и однозначного соответствия между поведением диэлектрической проницаемости и характеристиками динамики доменов, например, периметра доменных границ, как это показано в [3]. Известно, что длительное облучение кристаллов TGS микроволнами на частоте $40 \mathrm{GHz}$ сильно влияет на поведение низкочастотной диэлектрической проницаемости вблизи фазового перехода, указывая на определенную связь релаксационных механизмов поляризации на сверхвысоких (десятки гигагерц) и низких (килогерцы) частотах [4]. Поэтому была поставлена задача исследовать и проанализировать температурную динамику доменной структуры в ее связи с температурным поведением как микроволновой диэлектрической проницаемости, так и подавления аномалии диэлектрической проницаемости длительным микроволновым облучением. Экспериментальные исследования in situ температурной динамики доменной структуры кристалла TGS методами атомносиловой микроскопии (AFM) и диэлектрической спектроскопии в широкой области частот от $10^{1}$ до $10^{11} \mathrm{~Hz}$ вблизи фазового перехода проведены в условиях высокой стабилизации температуры образца и выдержке во времени в каждой точке измерений.

В работе [5] нами была сделана попытка проанализировать роль проводимости в сегнетоэлектрическом фазовом переходе с помощью диэлектрических спектров, основываясь на данных о проводимости доменных границ в кристалле TGS, полученных с помощью контактной сканирующей резистивной микроскопии. В продолжение начатых исследований предложены теоретические модели диэлектрического отклика на высокочастотное поле и развит подход к интерпретации диэлектрических спектров, основанный на представлении о значительном вкладе в диэлектрические спектры диффузионного движения протонов и, особенно, направленной диффузии (проводимости) подсистемы, объясняющий ряд наблюдаемых особенностей их поведения в области фазового перехода.

\section{2. Экспериментальные установки и методики измерений}

Кристаллы TGS (номинально чистые) $\left(\mathrm{N}^{+} \mathrm{H}_{3} \mathrm{CH}_{2} \mathrm{COOH}\right)_{2}\left(\mathrm{~N}^{+} \mathrm{H}_{3} \mathrm{CH}_{2} \mathrm{COO}^{-}\right) \mathrm{SO}_{4}^{2-}$ были выращены в Институте кристаллографии РАН методом изотермического испарения при температуре, меньшей температуры фазового перехода $T_{c}$. Перед проведением экспериментов по диэлектрическим измерениям кристаллы сначала отжигали при $T=110^{\circ} \mathrm{C}$ в течение двух часов. 
Bce эксперименты по подготовке и прецизионному исследованию поверхности образцов методом AFM проводили в контрольно-измерительном комплексе „TRACKPORE ROOM-05“, класс чистоты 8 ИCO(100), в составе которого имеется сканирующий зондовый микроскоп NTEGRA Prima (компания НT-МДТ, Зеленоград). Точность поддержания влажности в чистой зоне в диапазоне $30-70 \%$ составляет $\pm 1 \%$. Точность поддержания температуры в диапазоне $25 \pm 5^{\circ} \mathrm{C}$ составляет $\pm 0.05^{\circ} \mathrm{C}$. Поверхность естественного скола образцов изучали при относительной влажности 35\%. Для исследования доменной структуры использовали электрическую модификацию метода AFM - микроскопию пьезоэлектрического отклика (PFM) при нагреве и охлаждении образца в области температур от $38^{\circ} \mathrm{C}$ до температуры Кюри $\left(T_{c}=49.5^{\circ} \mathrm{C}\right)$. Все эксперименты проводили в условиях высокой стабилизации температуры образца и выдержке во времени в каждой точке измерений. Использовали стандартные кремниевые кантилеверы марки С21, балка В с покрытием Pt фирмы Mikroscience.

Измерение диэлектрических спектров, а именно, температурно-частотных зависимостей действительной $\left(\varepsilon^{\prime}\right)$ и мнимой $\left(\varepsilon^{\prime \prime}\right)$ частей диэлектрической проницаемости $\varepsilon$, проводили в диапазоне частот $(f)$ от $10^{1}$ до $10^{11} \mathrm{~Hz}$ и температур от комнатной до температуры фазового перехода. Измерения проводили при ступенчатом увеличении температуры (шаг по температуре изменялся от 1 до $0.1^{\circ}$ по мере приближения к $T_{c}$ ) с высокой степенью стабилизации в каждой точке. Время установления температуры, особенно вблизи фазового перехода, составляло один час и более. Для разных диапазонов частот использовали три установки. На низких частотах (до $10^{6} \mathrm{~Hz}$ ) измерения проводили методом импедансной спектроскопии на установке Novoterm1200 с анализатором импеданса Alpha-AN. Комплексную диэлектрическую проницаемость монокристаллических образцов TGS измеряли при ступенчатом нагреве в условиях стабилизации температуры в интервале $23-60^{\circ} \mathrm{C}$. Образцы вырезали из кристалла в виде плоскопараллельных пластин размером $5 \times 5 \mathrm{~mm}$ и толщиной $1 \mathrm{~mm}$, в которых большие грани ориентировали перпендикулярно оси $Y$. В качестве электродов использовали серебряную пасту.

В микроволновом диапазоне в зависимости от исследуемого диапазона частот использовали разные методики. На частотах $10-50 \mathrm{GHz}$ диэлектрическую проницаемость измеряли волноводным методом в режиме короткого замыкания. Образец кристалла толщиной $4 \mathrm{~mm}$, вырезанный по размеру волновода $\left(2.6 \times 5.2 \mathrm{~mm}^{2}\right)$, размещался у короткозамкнутого конца волновода, и изменение распределения поля в волноводе с кристаллом фиксировали по отношению к ненагруженному волноводу [6]. В частотном диапазоне $90-150 \mathrm{GHz}$ измерения $\varepsilon$ проводили на субмиллиметровом спектрометре, построенном на лампе обратной волны [7]. Перестраиваемое по частоте монохроматическое излучение распространялось в свободном пространстве в виде пучка диаметром $40 \mathrm{~mm}$, формируемого диэлектрическими линзами, и падало нормально на плоскость образца сечением $10.70-12.43 \mathrm{~mm}^{2}$ и толщиной $5.22 \mathrm{~mm}$. Прошедшее через образец излучение регистрировалось в оптико-акустическом приемнике. Расчет спектров $\varepsilon_{m}^{\prime}(\omega)$ и $\varepsilon_{m}^{\prime \prime}(\omega)$ проводили с помощью программного обеспечения „Epsilon 2001“.

\section{3. Экспериментальные результаты}

3.1. Атомно-силовая микроскопия. При нагревании образца выше $50^{\circ}$ и последующем охлаждении до комнатной температуры на PFM-изображениях наблюдается активная перестройка доменной структуры. Процессы при нагревании и охлаждении схожи, но пик активности при нагревании сдвинут в сторону более высоких температур. На рис. 1 приведены PFМ-изображения доменной структуры, полученные при охлаждении кристалла от 50 до $40^{\circ}$. После перехода через $T_{c}$ проявляются мелкие ламинарные домены шириной $\sim 0.2 \mu \mathrm{m}$. При этом положительные и отрицательные домены зарождаются равномерно. При дальнейшем понижении температуры домены укрупняются, появляются линзовидные домены $\left(48.5^{\circ}\right)$, доля которых увеличивается при охлаждении до $45^{\circ}$. При последующем охлаждении до $40^{\circ}$ начинается укрупнение доменов с преобладанием одной доменной компоненты. Схожие процессы изменения доменной структуры в области фазового перехода наблюдали с помощью электростатической силовой микроскопии [8].

C помощью программного обеспечения SPIP 6.1.1 (Image Metrology) была проведена обработка изображений и проведен расчет отношения полного периметра доменных границ к площади изображения $(D)$, который характеризует плотность доменных стенок по отношению к заданной поверхности (рис. 2,a). Несмотря на схожесть общего хода кривых $D(T)$, полученных при нагреве и охлаждении, параметр $D$ при одной и той же температуре различается (примерно на порядок), т. е. имеет место гистерезис. Активная фаза перестройки доменной структуры при нагревании происходит при более высокой температуре, чем при охлаждении (различие $\sim 2^{\circ}$ ). Изменение скорости процесса доменообразования наблюдается в районе $46^{\circ}$.

На рис. 2,b приведен график зависимости средней ширины доменов $(W)$ при охлаждении от 46.5 до $40.5^{\circ}$. Можно видеть, что на кривой наблюдается минимум в области $42-43^{\circ}$, соответствующий области „аномального“ поведения диэлектрической проницаемости от температуры. Схожее поведение плотности доменов при нагревании образца в той же области температур $\left(44^{\circ}\right)$ отмечали в [8].

Таким образом, основываясь на данных AFM, можно выделить характерные температуры, при которых фиксируются изменения параметров доменной структуры в области фазового перехода.

1. $42-44^{\circ} \mathrm{C}$ - фаза неактивной перестройки доменов (небольшие изменения средней ширины доменов). 

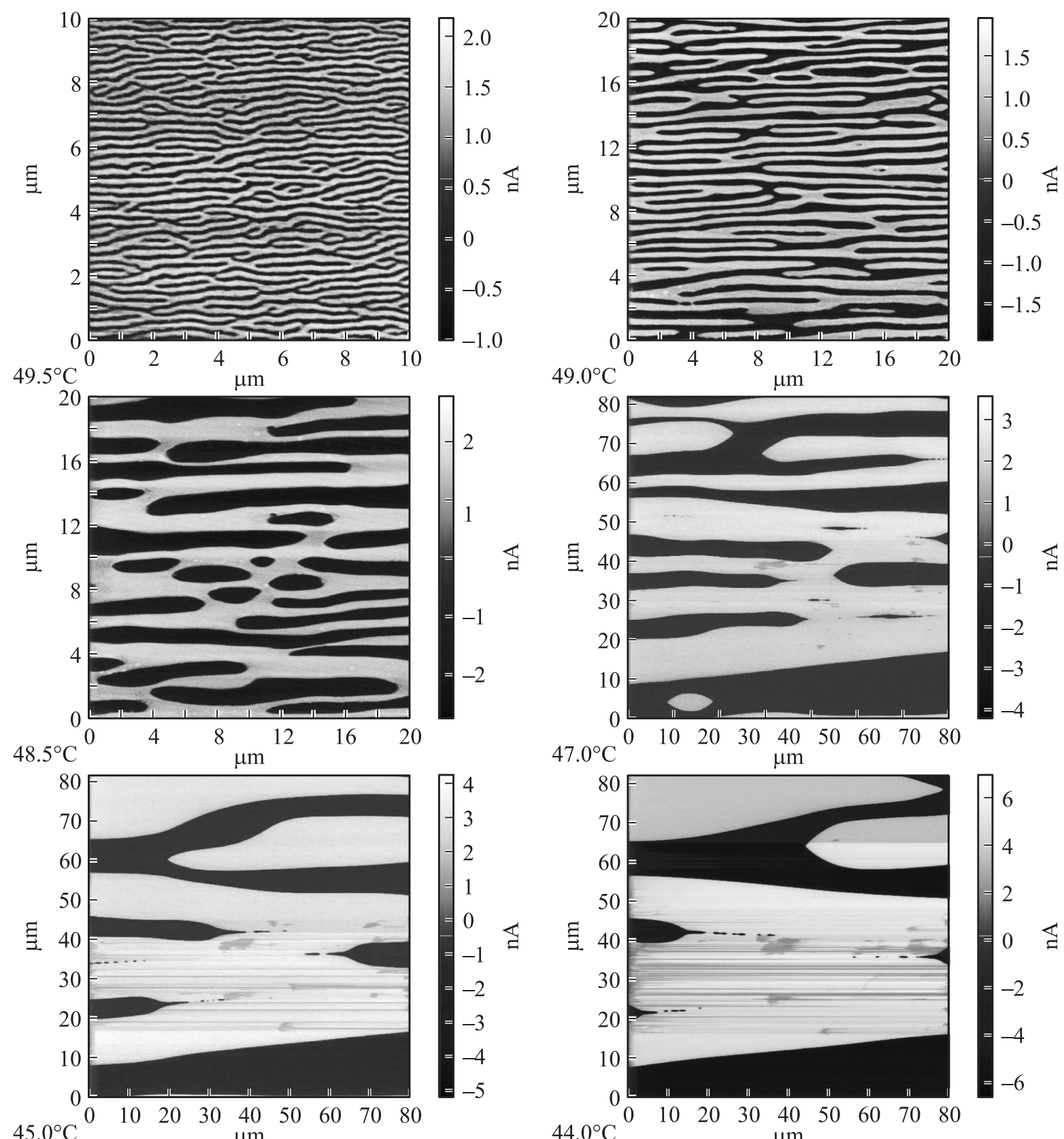
$47.0^{\circ} \mathrm{C}$
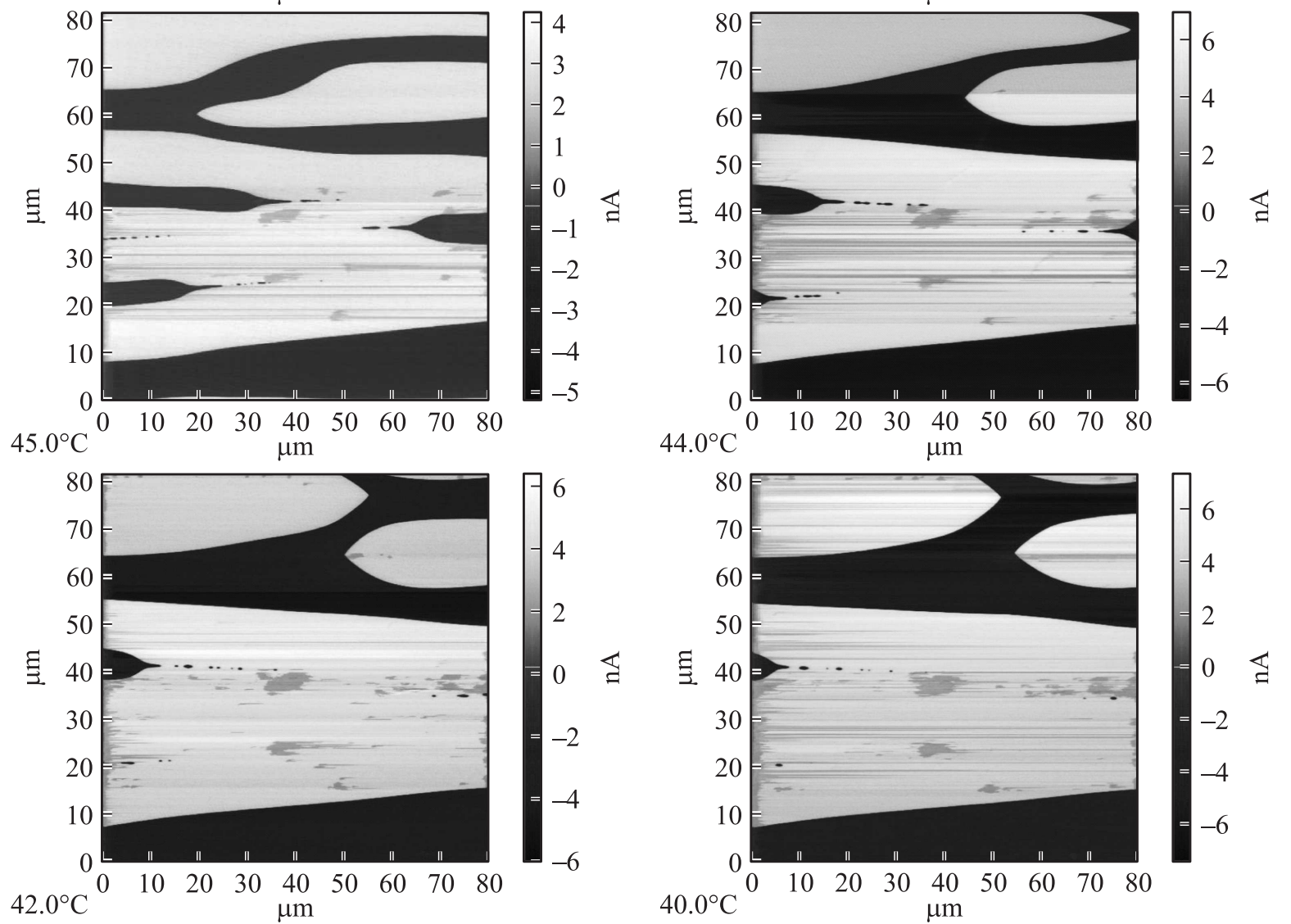

Рис. 1. РFM-изображения доменной структуры, полученные при охлаждении кристалла TGS от 49.5 до $40^{\circ} \mathrm{C}$. Полярная поверхность (010). Размер области сканирования $10 \times 10\left(49.5^{\circ} \mathrm{C}\right), 20 \times 20 \mathrm{~m}\left(49,48.5^{\circ} \mathrm{C}\right), 80 \times 80 \mathrm{~m} \quad\left(47-40^{\circ} \mathrm{C}\right) . \mathrm{nA}-$ электромеханический отклик, $\mathrm{nA}$. 
Соответствует узкому температурному интервалу, когда отсутствует подавление микроволнами аномалии низкочастотной диэлектрической проницаемости. Возможно, это указывает на наибольшее упорядочение доменной структуры перед ее разрушением.

2. $46^{\circ}$ - начало активной фазы перестройки доменов. Идет постепенный рост числа доменов, их продольное укрупнение при уменьшении поперечных размеров. Этот процесс происходит до тех пор, пока не образуется регулярная полосчатая структура, пронизывающая весь образец. Дальнейший рост температуры приводит уже к разрушению регулярной структуры, к ее измельчению в непосредственной близости и в самом фазовом переходе $\left(48-49.5^{\circ}\right)$.

3.2. Диэлектрическая спектроскопия. Диэлектрические спектры кристалла TGS, измеренные на низких частотах от $10^{1}$ до $10^{6} \mathrm{~Hz}$, при температурах от комнатной до температуры фазового перехода, при-
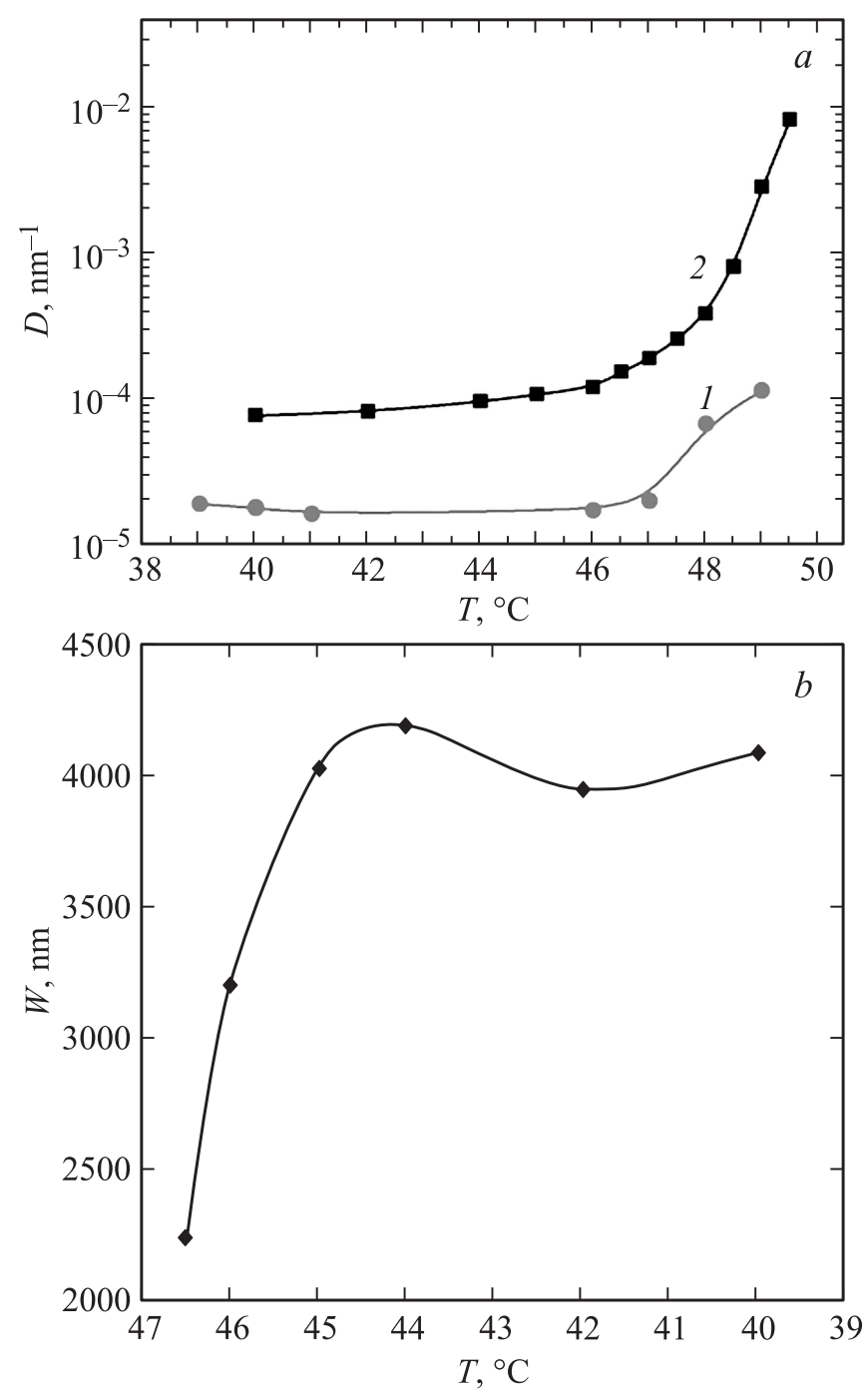

Рис. 2. Графики зависимости плотности доменов $(D)$ от температуры $T$, полученные при нагреве $(1)$ и охлаждении (2) кристалла TGS $(a)$, ширины доменов $W(T)$ при охлаждении от 46.5 до $40.5^{\circ} \mathrm{C}(b)$.
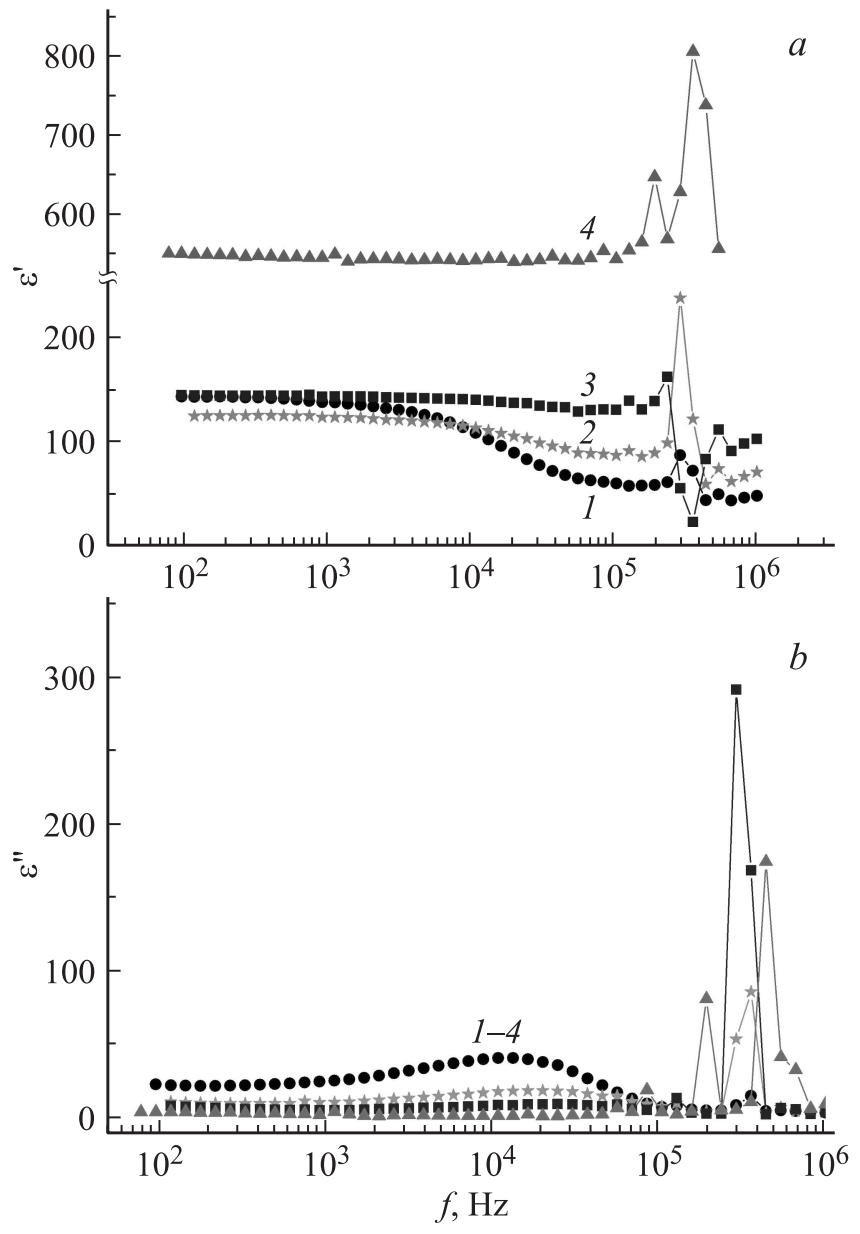

Рис. 3. Экспериментальные частотные зависимости $\varepsilon^{\prime}(a)$ и $\varepsilon^{\prime \prime}(b)$ при разных температурах $T: 1-24,2-36,3-42$, $4-49^{\circ} \mathrm{C}$. Нумерация кривых на $(b)$ идет сверху вниз.

ведены на рис. 3. Характерными особенностями этих спектров являются: 1) наличие низкочастотной релаксационной диэлектрической дисперсии на частотах вплоть до $10^{5} \mathrm{~Hz}$ при $24^{\circ} \mathrm{C} \leq T \leq 42^{\circ} \mathrm{C}$ и ее отсутствие при более высоких температурах, включая $\left.T_{c} ; 2\right)$ присутствие в диэлектрическом отклике колебательной компоненты на одной и той же частоте $3 \cdot 10^{5} \mathrm{~Hz}$ при всех исследованных температурах, с явным увеличением амплитуды отклика при подходе к $T_{c}$.

На рис. 4 представлены диэлектрические спектры, измеренные во всем исследованном диапазоне, включая микроволновый, при двух характерных температурах: $24^{\circ} \mathrm{C}\left(\right.$ a) и $46^{\circ}($ b $)$. При $T=24^{\circ} \mathrm{C}$ микроволновые диэлектрические спектры подобны низкочастотным, а спектры при $T=46^{\circ} \mathrm{C}$ обнаруживают особенности, которые не характерны ни для низкочастотных спектров, ни для релаксационного диэлектрического отклика вообще. Начиная с $f=10 \mathrm{GHz}$ значение $\varepsilon^{\prime}$ резко падает, опускаясь ниже уровня $\varepsilon_{\infty}$ при $f=40 \mathrm{GHz}$.

3.3 Теоретические модели и интерпретация диэлектрических спектров. Обсуждение экспериментальных диэлектрических спектров сегне- 

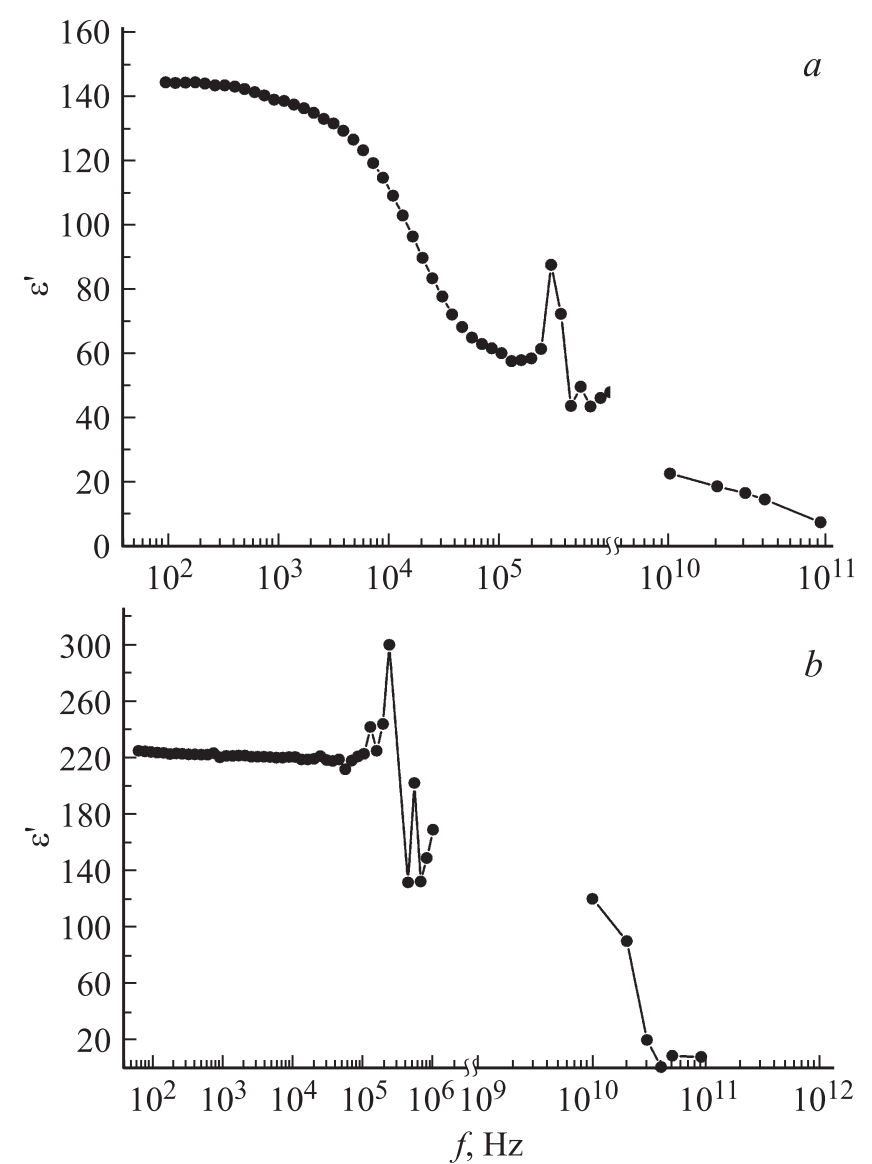

Рис. 4. Экспериментальные частотные зависимости $\varepsilon^{\prime}$ в диапазоне частот, включая микроволновой, для температур $24^{\circ} \mathrm{C}(a)$ и $46^{\circ} \mathrm{C}(b)$.

тоэлектрического кристалла TGS проведем, основываясь на двух модельных представлениях. Во-первых, будем считать, что в диэлектрическом отклике присутствуют одновременно два механизма дисперсии: 1) релаксационный ориентационный, характерный для диэлектриков, и 2) связанный с наличием сквозной (не зависящей от времени) статической проводимости. Во-вторых, воспользуемся моделью динамической проводимости, разработанной для суперионных проводников [9] при описании зависящей от времени проводимости.

В первом представлении диэлектрический отклик ориентационной составляющей описывается формулой из модели Дебая вращения диполей в вязкой среде, где зависимость диэлектрической проницаемости $\varepsilon$ от частоты $\omega$ имеет вид (1)

$$
\varepsilon^{*}=\varepsilon_{\infty}+\frac{\varepsilon^{\prime}(0)-\varepsilon_{\infty}}{1+i \omega \tau_{1}}
$$

Диэлектрический отклик проводящей составляющей $\sigma$ описывается формулой по модели Друде для движения носителей заряда в вязкой среде

$$
\sigma^{*}=\frac{\sigma_{0}}{1-i \omega \tau_{2}} .
$$

Здесь $\varepsilon^{\prime}(0)$ и $\varepsilon_{\infty}-$ статическая и высокочастотная составляющие диэлектрической проницаемости, соответственно, $\sigma_{0}-$ не зависящая от частоты проводимость, $\tau_{1}$ и $\tau_{2}$ - времена релаксации в моделях Дебая и Друде, соответственно. Связь между $\varepsilon^{*}$ и $\sigma^{*}$ дается классическим выражением

$$
\varepsilon^{*}=\frac{-i \sigma^{*}}{\varepsilon_{0} \omega} .
$$

После разделения действительных и мнимых частей уравнений (1)-(3) получаем для $\varepsilon^{\prime}$ и $\varepsilon^{\prime \prime}$ частотные зависимости вида

$$
\begin{gathered}
\varepsilon^{\prime}(\omega)=\varepsilon_{\infty}+\frac{\varepsilon^{\prime}(0)-\varepsilon_{\infty}}{1+\omega^{2} \tau_{1}^{2}}-\frac{\sigma_{0} \tau_{2}}{\varepsilon_{0}\left(1+\omega^{2} \tau_{2}^{2}\right)}, \\
\varepsilon^{\prime \prime}(\omega)=\frac{\left(\varepsilon^{\prime}(0)-\varepsilon_{\infty}\right) \omega \tau_{1}}{1+\omega^{2} \tau_{1}^{2}}+\frac{\sigma_{0}}{\varepsilon_{0} \omega\left(1+\omega^{2} \tau_{2}^{2}\right)} .
\end{gathered}
$$

Из выражений (4) и (5) следует, что вклад каждого из механизмов в диэлектрический отклик действительной и мнимой частей диэлектрической проницаемости различен: вклады обоих механизмов в $\varepsilon^{\prime \prime}$ всегда положительны, а в $\varepsilon^{\prime}$ они входят с разными знаками, поэтому $\varepsilon^{\prime}$ может быть как отрицательным, так и положительным.

Вдали от областей дисперсии $(\omega \tau<1)$, где диэлектрическая проницаемость не зависит от частоты, знак $\varepsilon^{\prime}(\omega)$ всегда будет определяться преобладающим вкладом основного механизма диэлектрического отклика, и наблюдение небольшого вклада неосновного механизма, даже с противоположным знаком, может быть трудной задачей, если нет других доказательств его присутствия в спектре. В областях дисперсии $(\omega \tau \sin 1)$, где вклад основного механизма падает с частотой, присутствие неосновного механизма может при определенных параметрах обоих механизмов дисперсии проявлять себя в виде максимума или минимума на фоне убывающего вклада основного механизма дисперсии.

Этот подход был использован в [5] и было показано, что наличие только статической проводимости не может объяснить все особенности наблюдаемых диэлектрических спектров. Поэтому в данной работе для анализа спектров во всем частотном диапазоне, включая область резонансного отклика и микроволновую область, была использована модель динамической, т. е. зависящей от частоты, проводимости.

Феноменологическая модель динамической проводимости, представленная в [9], была разработана для расчета диэлектрических спектров суперионных проводников и описывала температурную динамику подрешетки, элементы которой с ростом температуры начинают совершать диффузионное движение. До сих пор эта теория не применялась для анализа спектров сегнетоэлектриков. Считалось, что проводимость в них, если и имеет место, то недостаточна, чтобы оказывать влияние на диэлектрический отклик этих материалов. Это отчасти верно, если под проводимостью иметь в виду только сквозную проводимость. 

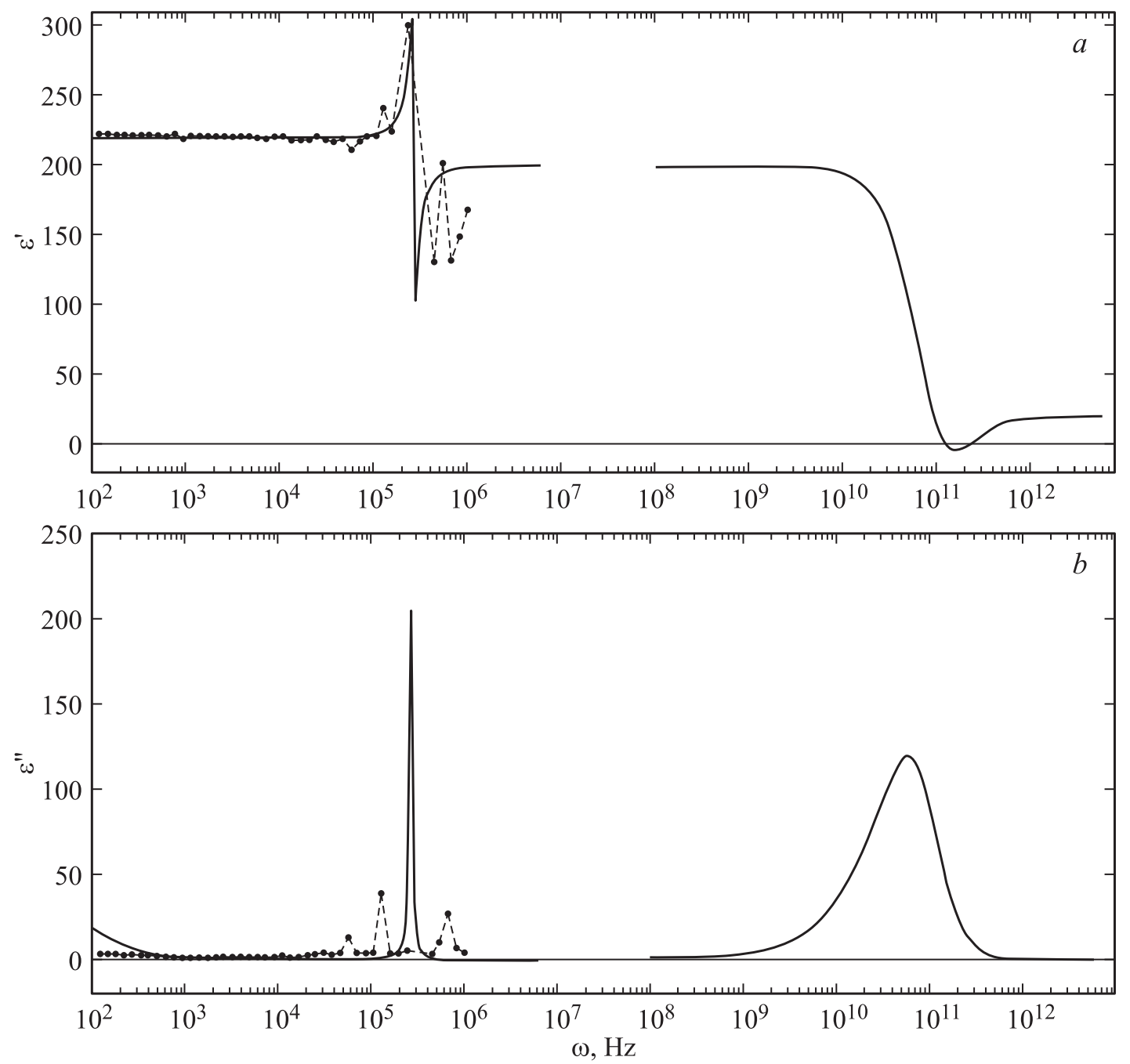

Рис. 5. Диэлектрические спектры двух областей дисперсии $\varepsilon^{\prime}(a)$ и $\varepsilon^{\prime \prime}(b)$ при $T=46^{\circ} \mathrm{C}$ : расчет - сплошные линии, эксперимент - штриховые линии.

В модели динамической проводимости в качестве основных, изменяющихся с температурой параметров, присутствуют: частота осцилляций $\omega_{0}$, связанное с основной кристаллической решеткой затухание $\gamma$, и время локализации диффундирующих частиц $\tau_{R}$. Изменяя время локализации от больших значений, соответствующих осцилляторному отклику, до очень малых $\left(\tau_{R}=0\right)$, соответствующих отклику Друде, можно проследить за температурным разупорядочением системы. Кроме того, изменяя затухание, можно привести систему к отклику дебаевского типа. Полученное в [9] в комплексном виде выражение для проводимости нами преобразовано в выражение для диэлектрической проницаемости:

$$
\begin{aligned}
& \varepsilon^{\prime}(\omega)=-4 \pi \frac{n e^{2}}{m} \\
& \times \frac{\gamma^{2}\left(1+\omega^{2} \tau_{R}^{2}\right)+\omega_{0}^{2} \gamma \tau_{R}\left(1-\gamma \tau_{R}\right)}{\varepsilon_{0}\left(\left(\gamma \tau_{R}\left(\omega_{0}^{2}-\omega^{2}\right)+\left(\gamma^{2}-\omega_{0}^{2}\right)\right)^{2}+\left(\omega \gamma\left(1+\gamma \tau_{R}\right)\right)^{2}\right)},
\end{aligned}
$$

$$
\begin{aligned}
& \varepsilon^{\prime \prime}(\omega)=4 \pi \frac{n e^{2}}{m} \\
& \times \frac{\gamma\left(\gamma^{2}\left(1+\omega^{2} \tau_{R}^{2}\right)-\omega_{0}^{2}\left(1-\gamma \tau_{R}\right)\right)}{\varepsilon_{0} \omega\left(\left(\gamma \tau_{R}\left(\omega_{0}^{2}-\omega^{2}\right)+\left(\gamma^{2}-\omega_{0}^{2}\right)\right)^{2}+\left(\omega \gamma\left(1+\gamma \tau_{R}\right)\right)^{2}\right)} .
\end{aligned}
$$

Итак, модель динамической проводимости была применена для описания всех трех наблюдаемых в эксперименте дисперсий: дебаевской, осцилляторной и микроволновой. На рис. 5 представлены кривые, рассчитанные для двух областей дисперсии при $T=46^{\circ}$. Низкочастотная дисперсия на частоте $f=10 \mathrm{kHz}$ при этой температуре, как отмечалось выше, уже отсутствует, а при более низких температурах хорошо описывается моделью со статической проводимостью [5]. Для рассматриваемых двух других областей дисперсии на частотах $f=300 \mathrm{kHz}$ и $f=40 \mathrm{GHz}$ используются формулы (6) и (7) с параметрами $f_{0}=270 \mathrm{kHz} ; \tau_{R}=10^{-2} \mathrm{~s}$, $\gamma=25 \mathrm{kHz}$ для осцилляторного диэлектрического от- 
клика и $f_{0}=94 \mathrm{GHz} ; \tau_{R}=10^{-8} \mathrm{~s}, \quad \gamma=178 \mathrm{GHz}$ для микроволнового. По этим параметрам были рассчитаны кривые, согласующиеся с экспериментальными зависимостями. Как видно, и действительная (рис. 5,a), и мнимая (рис. 5,b) части диэлектрической проницаемости хорошо описываются в рамках предложенного механизма.

\section{4. Заключение}

Предложенные теоретические модели, учитывающие вклад в диэлектрические спектры проводимости, и расчет спектров на их основе позволили достичь хорошего согласования с полученными экспериментальными данными, в том числе и микроскопическими исследованиями динамики доменной структуры и проводимости доменных границ. Важной особенностью предложенного подхода к анализу связи доменной структуры с макроскопическими характеристиками, в частности, с диэлектрической проницаемостью, служит тот факт, что существует связь между низко- и высокочастотным диэлектрическими откликами, что указывает на зависимость низкочастотной релаксационной поляризации от процессов, определяющих диэлектрический отклик на сверхвысоких частотах.

Диэлектрические спектры, снятые в широком диапазоне частот от 10 до $10^{11} \mathrm{~Hz}$, несут информацию о проводимости изучаемой среды. Это означает, что в среде имеет место как статическая, так и динамическая проводимости. Диффузионные процессы играют значительную, если не основную, роль в диэлектрическом отклике водородсодержащих сегнетоэлектриков.

Совместное использование структурно-чувствительных методов (атомно-силовой микроскопии и диэлектрической спектроскопии) представляется хорошим инструментом для изучения физических процессов в водородсодержащих сегнетоэлектриках, что перспективно для контроля качества материалов в технике сверхвысоких частот.

\section{Список литературы}

[1] Ф.М. Лотонов, В.К. Новик, Н.Д. Гаврилова. Вестн. МГУ. Cep. 36 5, 27 (2006).

[2] C. Barbecaru. Phys. Status Solidi C 7, 5, 1440 (2010).

[3] С.Н. Дрождин, О.М. Голицина. ФТТ 54, 853 (2012).

[4] Г.И. Овчинникова, А.Н. Солошенко, Н.В. Белугина, Р.В. Гайнутдинов, А.Л. Толстихина. Поверхность 10, 24 (2009).

[5] Г.И. Овчинникова, И.Ю. Полякова, Е.С. Иванова, Р.В. Гайнутдинов, Н.В. Белугина, А.Л. Толстихина, В.В. Гребенев. ФTT 57, 531 (2015).

[6] Г.И. Дьяконов. Автореф. канд. дис. МГУ, М. (1985). 24 с.

[7] S.V. Danilova, E.S. Ivanova, A.K. Malyshkin, G.I. Ovchinnikova, Yu.A. Pirogov. Phys. Wave Phenom. 21, 231 (2013).

[8] E.Z. Luo, Z. Xie, J.B. Xu, I.H. Wilson. Phys. Rev. B 61, 203 (2000).

[9] А.А. Волков, Г.В. Козлов, С.П. Лебедев, А.С. Ракитин. ФТТ 32, 329 (1990). 